\title{
Optical readout of the intracellular environment using
}

\section{nanoparticle transducers}

\author{
Annette Dowd ${ }^{1}$, Dakrong Pissuwan ${ }^{2}$, Michael B. Cortie ${ }^{1}$
}

${ }^{1}$ Institute for Nanoscale Technology, University of Technology Sydney, PO Box 123, Broadway NSW

2007, Australia

${ }^{2}$ Material Science and Engineering Program, Multidisciplinary Unit, Mahidol University, Bangkok, 10400, Thailand

Corresponding author: Dowd, A. (annette.dowd@uts.edu.au)

There is rapid growth in the use of multi-functional nanoparticles as transducers to probe the intracellular environment. New designs of nanoparticles can provide quantitative information at sub-cellular resolution on parameters such as $\mathrm{pH}$, temperature and concentration of nicotinamide adenine dinucleotide (NADH) or selected metal ions. This new work builds on the existing practice of using nanoparticles and fluorescent dyes to provide enhanced microscopic images of cells, but goes beyond it by adding new functionalities and analytical capabilities. Here we review the recent literature on the development of such nanoparticles for simultaneous biosensing and imaging. We explore and examine the different measurements that will be possible and analyze the likely accuracy and resolution that could be achieved.

Keywords: intracellular environment, nanoparticle sensor, sub-cellular transduction 


\section{Biosensing using nanoparticles}

Nanoparticles with multi-functional properties can now be designed to transduct (see Glossary) biochemical signals at sub-cellular spatial resolution. Solid nanoparticles offer several advantages over fluorescent dyes when imaging and probing the intracellular environment. For example, nanoparticles do not bleach as readily as fluorescent dye probes, they can be readily internalized into cells $[4,5]$, many appear to have low cytotoxicity [6, 7], some can capture incident light and amplify the local electromagnetic field, and, of course, they have a massive surface-to-volume ratio and so a very small quantity can have a surprisingly large effect. An early application of nanoparticles was to produce surface enhanced Raman scattering (SERS). Under suitable conditions it becomes possible to detect an analyte at zeptomolar levels [8]. The application of SERS within live cells has also been investigated for some years and information on this topic is widely available $[9,10]$. There are, however, a number of other, lesser known, ways in which a nanoparticle can be used to transduct biochemically-relevant parameters such as intracellular temperature, $\mathrm{pH}$, or the concentration of metabolites or metal ions. These are the focus of the present review.

\section{Transduction of intracellular temperature}

The temperature difference between the intra- and extracellular environment, or within the cell, can provide a sensitive indication of cellular activity [11] and how it might be changed by drugs or disease [12]. Under static conditions, objects the size of a typical cell will rapidly reach thermal equilibrium in an aqueous environment. However, exo- or endothermic processes within cells (such as metabolism or 
division) can perturb the intracellular temperature slightly. An obvious measurement strategy is to insert a very small thermocouple into the cell, which can give a precision of $<0.1^{\circ} \mathrm{C}[13]$ while insertion of a microscopic mechanical resonator can give a sensitivity of $\sim 0.002^{\circ} \mathrm{C}$, equivalent to the $5 \mathrm{pJ}$ heat output of a single brown fat cell [14]. Unfortunately, a cell will almost certainly be significantly perturbed or damaged by the above techniques which are also rather difficult to set-up. In contrast, photoacoustic thermometry can provide a precision of about $0.2{ }^{\circ} \mathrm{C}$ [15], which is similar to the 0.1 to $0.8^{\circ} \mathrm{C}$ precision obtained from fluorescent dyes of various kinds $[11,16]$. The activity of temperature-sensitive enzymes is another possible probe, and this gives a precision of about $0.7^{\circ} \mathrm{C}$ [17]. Unfortunately, the latter two transduction modalities are also sensitive to the $\mathrm{pH}$ and ionic strength of the intracellular environment, both of which are variable. Recently, however, reports have emerged of new designs of nanoparticles that are capable of transducting the intracellular temperature. The transduction of the temperature can be combined with imaging of the nanoparticle to generate spatial information.

The expansion of the crystal lattice of a quantum dot (QD) (see Box 1) due to an increase in temperature will cause a red-shift in its emission peak, and this has been used to characterize inhomogeneous temperature distributions within live cells. For example Yang et al. [18] used commercial QDs to investigate the effect of $\mathrm{Ca}^{2+}$ additions on the temperature of $\mathrm{NIH} / 3 \mathrm{~T} 3$ cells. A precision of about $0.3^{\circ} \mathrm{C}$ seems to have been obtained. In general, however, blinking of QDs could be a problem because it might disrupt a sequence of measurements [19].

Measurement of intracellular temperatures by probing the electronic spin state of defects within nano-diamonds was reported recently by Kucsko et al. [20] A precision of better than $0.05{ }^{\circ} \mathrm{C}$ was obtained and the authors note that a precision 
approaching $0.001^{\circ} \mathrm{C}$ should be achievable in theory. The electronic spins within the nitrogen-vacancies are first manipulated using microwave radiation and then probed using electron spin resonance (ESR) spectroscopy, Figure 1. The elastic strain around the $\mathrm{N}-\mathrm{V}$ centres of the nanodiamond are influenced by temperature and it is this that causes the spin signal to vary.

A temperature-sensitive dye can still be safely used to monitor intracellular temperature provided that it can be chemically isolated from the intracellular environment. For example, europium thenoyltrifluoroacetonate can be encapsulated within a poly(methyl methacrylate) (PMMA) nanoparticle [19]. Delivery of the hybrid construct in this case was assisted by coating it with the cationic polymer poly(allylamine hydrochloride) (PAH).

Another example of a hybrid scheme may be found in conjugates of QD and dyes [21]. Here a temperature sensitive-dye (cyanine) is conjugated to a rod-shaped QD of CdS. The change in temperature is not inferred directly from the dye or from the QD, but rather from the FRET process (See Box 2) occurring between dye and QD. Estimates of temperature are made by taking the ratio of emission intensities at specific wavelengths. A precision of at least $0.2{ }^{\circ} \mathrm{C}$ was demonstrated. A further example of using a fluorophore somewhat indirectly is provided by Okabe et al. [22]. Here fluorescent molecules are inserted into a nanoparticle of temperatureresponsive poly-N-n-propylacrylamide (pNNPAM). The latter has the property that it is more permeable to its aqueous environment at lower temperatures, becoming increasingly less so as the temperature rises above $25^{\circ} \mathrm{C}$. Ingress of aqueous ions at low temperatures quenches the fluorophore, but as the temperature rises the polymer contracts, expelling water and resulting in a systematic increase of fluorescence. The precision is about $0.5^{\circ} \mathrm{C}$. 


\section{Metabolites}

Intracellular metabolite levels and dynamics reflect the cellular response to changes in the environment or genetic expression. Monitoring the concentration of these molecules, especially with sufficient spatial resolution to resolve cell-to-cell or intracellular variation is of scientific importance for understanding cellular behavior and has practical application in the study of disease and drug treatment. A number of different metabolites have been investigated with nanoparticles including ATP [23], cysteine [24] and complex 1 [25]. In this review we focus on detection and imaging of the two intensely studied molecular species of most biological importance, ROS and $\mathrm{NADH}$.

\section{Optical imaging of intracellular ROS}

Reactive oxygen species (ROS) in the intracellular environment come from normal cellular metabolism or exogenous sources. ROS are used in cell regulation and signaling (e.g. apoptosis) and their concentration is tightly controlled by antioxidant and enzymatic molecular activity. Pathologies associated with ROS include chronic diseases and cancer. Optical imaging using fluorescent dyes has been used [26], however possible drawbacks of this technique include lack of localization at the site of the redox reaction, non-specific reactions during delivery of the dyes to target tissues/organelles, photobleaching [27] and cytotoxicity [28]. To overcome these issues, nanoparticles have also been considered as probes [2]. Of course, some means must be found to use the nanoparticle to transduct a signal proportional to ROS concentration and the most obvious strategy is to conjugate the nanoparticle with a ROS-sensitive fluorophore. In this way, the nanoparticle can be exploited to 
selectively deliver the fluorophore to a target location, while the fluorophore provides a signal in proportion to the ROS [2]. As before, examination of the cell is conveniently achieved using a confocal microscope.

One example of this idea is a silicate sol-gel nanoprobe which contains the fluorescent dye 5(6)-carboxyfluorescein [29]. Encapsulation of the dye within the inorganic host reduces the leaching of fluorophores and hence minimizes cell toxicity. ROS concentrations as low as $1 \mathrm{nM}$ could be detected. Alternatively, chemical changes induced by the ROS can be used to trigger a fluorescent signal. Kim et al. [30] showed how hyaluronic acid (HA) conjugated with chlorin e6 (Ce6) could be used as an indicator of ROS production in stimulated macrophage cells. The presence of ROS causes degradation of the HA [31, 32] which releases fluorescent Ce6.

Gold nanoparticles (GNPs) are an alternative platform for detection of intracellular ROS. Lee et al. synthesized a ROS-sensitive probe by immobilizing fluorescein-HA conjugates on the surface of GNPs [33]. The key point here is that while the fluorophore is on or close to the surface of the GNP its fluorescence is quenched (See Box 1). The presence of ROS releases the fluorophore which can then escape the proximity of the GNP and become fluorescent. When live macrophages were treated with such conjugates and stimulated by lipopolysaccharide, ROS production occurred, resulting in a strong fluorescent signal. The generation of ROS (and its location within the macrophages) could be monitored using confocal microscope analysis, Figure 2.

The situation for very small $(<2 \mathrm{~nm})$ gold nanoparticles is subtly different. In this case the particle and its stabilizing ligand can be thought to form a single hybrid entity 
which is luminescent by virtue of its electronic configuration. Reaction with ROS can alter the surface electronic states of the cluster and change its properties. $\mathrm{H}_{2} \mathrm{O}_{2}$ can be detected, for example, by the effect it has on quenching the fluorescence of the complex formed between $2 \mathrm{~nm}$ Au 'nanodots' and 11-mercaptoundecanoic acid [34].

Although most ROS-sensing nanoparticles make use of fluorescence, there are techniques for the transduction of a chemical environment even without a fluorophore. SERS (see Box 2) is one of the mostly widely known of these possibilities [2, 4]. In this context, Au nanoshells have been used for measuring reductive and oxidative stress in fibroblast cells. The SERS probe molecules were 1,8-diaza-4,5-dithia-1,8-di(2-chloro-[1,4]-naphthoquinone-3-yl)octane and 2mercapto-benzene-1,4-diol. These molecules have redox-active quinone moieties which gain or lose $\mathrm{H}^{+}$ions with a detectable change in their Raman spectra thereby allowing the detection of intracellular redox potentials without using fluorescent dyes [35].

The detection of dissolved oxygen is a related issue to the detection of ROS. Some nanoparticle-based schemes for $\mathrm{O}_{2}$ detection are discussed in recent reviews [36, 37]. More recently polymer core-shell particles with an oxygen-sensitive platinum porphyrin dye (PtTFPP) covalently attached to the hydrophobic core have been examined [38]. These nanoparticles were taken up by Vero (monkey) cells, resided in organelles in the cytoplasm and showed qualitatively decreasing fluorescence as atmospheric $\mathrm{O}_{2}$ was decreased from $20 \%$ to $0 \%$. In other recent work, quantitative analyses were achieved by including a second $\mathrm{O}_{2}$-insensitive reference fluorophore which acts as a fluorescence energy transfer (FRET) donor (see Box 2) for PtTFPP [39]. Further improvement of this scheme to obtain sub-cellular resolution would be of great interest. 


\section{Optical probes for NADH}

$\mathrm{NADH}$ is involved in multiple biological processes so its concentration reflects cellular redox environment, mitochondrial activity, oxidative stress, and metabolism [40]. It can be detected by a variety of means including UV-induced autofluorescence (which unfortunately has low sensitivity and induces damage to the cell) and a fluorescent protein which provides a high detection specificity in living cells [41]. In addition, NADH is a strong reductant, and this can be exploited in analyses. Zhang et al. have reported a scheme that exploits the reduction of $\mathrm{Cu}^{2+}$ to $\mathrm{Cu}^{0}$ by $\mathrm{NADH}$, with the $\mathrm{Cu}^{0}$ precipitating onto the surface of intracellular GNPs to form a Au@Cu core-shell particle [42]. Localized changes in NADH concentration could be detected from the dark-field spectra taken from individual nanoparticles using an optical microscope. As a demonstration of the technique, the metabolism of HeLa cells was slowed with taxol (an anti-cancer drug) thereby causing a decreased production of $\mathrm{NADH}$. Dark field spectra collected from individual nanoparticles within a cell permitted the formation and intracellular distribution of the NADH to be mapped, Figure 3.

Quantum dots (QDs) can be also used as an optical label for probing NADH. For example CdSe/ZnS QDs functionalized with Nile blue FRET acceptor were used to show that the addition of D-glucose to HeLa cells caused an increase in QD fluorescence when the Nile blue was reduced (due to production of NADH generated by glycolysis and the Krebs cycle) whereas there was no change with the addition of L-glucose (which is not a participant in the glycolysis pathway) [43]. 


\section{Detection of metal ions}

Metal ions are essential co-factors for many enzymes, often to catalyze redox reactions. There are a number of pathologies associated with reduced enzyme activity resulting from faulty metal ion regulation. A number of nanoparticle transducers for ion concentration have been investigated. In general these are based on modification of a fluorescent signal. The most commonly detected ion is $\mathrm{Cu}^{2+}$ but methods for determining the presence of $\mathrm{Zn}^{2+}, \mathrm{Ca}^{2+}, \mathrm{K}^{+}, \mathrm{Na}^{+}, \mathrm{Al}^{3+}, \mathrm{Fe}^{2+}, \mathrm{Mg}^{2+}, \mathrm{Pb}^{2+}$ $\mathrm{Cr}^{3+}, \mathrm{Hg}^{2+}$ and $\mathrm{UO}_{2}{ }^{2+}$ have also been reported [37, 44-47]. Specificity of the response must of course be verified because fluorescence is also affected by ions such as $\mathrm{H}_{2} \mathrm{PO}_{4}^{-}, \mathrm{H}^{+}$and $\mathrm{S}_{2}^{-}[45,48]$.

As an illustrative example of the technology, Durgadas et al. used fluorescent bovine serum albumin-coated Au nanoparticles conjugated to folic acid for improved internalization in HeLa cells [49]. The presence of $\mathrm{Cu}^{2+}$ within the cells quenched the red Au-BSA fluorescence. Similar $\mathrm{Cu}^{2+}$ fluorescence quenching was seen with BODIPY-functionalized $\mathrm{Au}$ nanoparticles in $\mathrm{Cu}^{2+}$ treated HeLa cells [50] and fluorescein-functionalized silica nanoparticles [51]. Unfortunately, the data obtained from a single channel measurement is generally not quantitative for $\mathrm{Cu}^{2+}$ concentration. Ratiometric measurements with dual emission nanoparticles have been more successful in this regard. For example, fixed permeabilized MCF-7 cells containing Rhodamine B-labelled carbon dots ('C-dots') localized in vesicles were incubated in $50 \mathrm{uM} \mathrm{Cu}^{2+}$ and the associated fluorescence ratio maps showed clear changes resulting from an increase in intracellular $\mathrm{Cu}^{2+}$ [52]. Intracellular $\mathrm{Cu}^{2+}$ 
changes were also imaged in HeLa cells after incubation in $4 \mathrm{uM} \mathrm{Cu}^{2+}$ with FITCdoped silica nanoparticles linked to RBITC [53]. These nanoparticles have the advantage of sensitivity down to $10 \mathrm{nM} \mathrm{Cu}^{2+}$. Zhu et al [54] used a combination of nanoparticles based on a red-emitting CdSe/ZnS quantum dot (as the reference signal) and a blue-emitting carbon dot functionalized with a specific $\mathrm{Cu}^{2+}$ receptor (AE-TPEA). Treatment with $\mathrm{Cu}^{2+}$ induced quenching of the $\mathrm{C}$-dot fluorescence but not of the reference, permitting a quantitative determination of $\mathrm{Cu}^{2+} \cdot \mathrm{Zn}^{2+}$ and $\mathrm{Ca}^{2+}$ have also been imaged using a ratiometric measurement from nanoparticles [48, 55].

\section{Measurement and mapping of $\mathrm{pH}$}

Intracellular $\mathrm{pH}$ affects a wide variety of intracellular processes including enzymatic activity, ion channel activity and cell cycle phase by changing the ionization state of peptides and proteins. For this reason intracellular $\mathrm{pH}$ is tightly controlled and deviations from the optimum range can indicate adverse changes in the cell's $\mathrm{pH}$ sensing or regulation mechanisms or its metabolic activity.

Optical transduction of cellular $\mathrm{pH}$ has been traditionally achieved using fluorescent or absorptive indicators. Unfortunately, in addition to the generic issues that plague the use of all free dyes, $\mathrm{pH}$ indicator dyes are also susceptible to interference from the binding of intracellular proteins [56]. Furthermore, fluorescence-based $\mathrm{pH}$ indicators generally operate over a very limited $\mathrm{pH}$ range, typically about one $\mathrm{pH}$ unit from the indicator $\mathrm{p} K_{\mathrm{a}}$. Nanoparticle-based $\mathrm{pH}$ transducers can overcome many of these limitations by encapsulating the indicator and limiting its interaction with 
biological molecules or by including a number of different dyes to extend the measurable $\mathrm{pH}$ range. A useful summary of the types of nanoparticles developed for optical transduction of $\mathrm{pH}$ is found in [57]. There are many reports on the use of nanoparticles to map intracellular (usually endosomal) $\mathrm{pH}$, however many of these do not present quantitative information about the mapped $\mathrm{pH}$, an omission that is rarely commented on. In this section we highlight recent successful application of nanoparticles to quantitative measurement and mapping of intracellular $\mathrm{pH}$.

For example, GNPs with two fluorophores $(\mathrm{pH}$-insensitive rhodamine and $\mathrm{pH}$ sensitive anthracene) were endocytosed by $\mathrm{CHO}$ cells. Fluorescence was excited with $364 \mathrm{~nm}$ radiation and the ratio of the two fluorescence bands was found to be correlated with the $\mathrm{pH}$ in the 4 to 8 range. A precision of $\pm 0.3 \mathrm{pH}$ unit was claimed, which is sufficient to differentiate various locations in the cell [58]. In another example, the $\mathrm{pH}$ of endosomes in HeLa cells was monitored with quantum dot (CdS/CdSe) - fluorophore (5(6)-carboxy-X-rhodamine) hybrids. In this case it is the linker molecule (DNA) that is sensitive to $\mathrm{pH}$ (via conformation change) and the modulation of the fluorescent signal is via FRET [59]. Imaging was straightforward: a single excitation wavelength $(460 \mathrm{~nm})$ generated two easily separated fluorescent bands centered on $560 \mathrm{~nm}$ and $610 \mathrm{~nm}$. The system produced a precision of \pm 0.4 unit in the $\mathrm{pH}$ range 6.0 to 7.4 .

Mesoporous silica nanoparticles can also be loaded with a pair of $\mathrm{pH}$-sensitive fluorophores such as fluorescein isothiocyanate (FITC) and Rhodamine B isothiocyanate (RITC). Chen et al [60] used such nanoparticles with negatively or positively charged surfaces to map the $\mathrm{pH}$ in HeLa cell endosomes. The endosomal $\mathrm{pH}$ reported by the negatively charged nanoparticles was $<5$, while that reported by the positively charged nanoparticles was in the range 6.0 to 6.3 . The difference is 
explained by assuming some of the positively charged nanoparticles have escaped the endosomes into the cytosol (which has a higher $\mathrm{pH}$ ) through a charge-mediated mechanism.

Although nanoparticles usually enter the cell by the endocytic pathway, accurate measurement of the $\mathrm{pH}$ over the lifetime of the vesicle is not straightforward because the evolution of $\mathrm{pH}$ can extend over more than two $\mathrm{pH}$ units. A focus of recent research has been to increase the measureable $\mathrm{pH}$ range. Tsou and co-workers extended the $\mathrm{pH}$ response of silica nanoparticles loaded with FITC and RITC by designing the nanoparticle to exploit the surface-curvature dependence of the fluorophore $p K_{a}$ [61]. These particles were used to determine the $\mathrm{pH}$ in predominantly endocytic regions of HeLa cells over pH from 4.5 to 7.5. Large 200 $\mathrm{nm}$ polymeric nanoparticles containing Rhodamine $6 \mathrm{G} \quad(\mathrm{R} 6 \mathrm{G})$ and 1,3phenylenediamine have been shown to have a useful $\mathrm{pH}$ range from 8.0 to 3.0 although their use it limited to situations where the $\mathrm{pH}$ monotonically decreases since increase in nanoparticle protonation results in irreversible R6G release [62]. Triple labelling of nanoparticles is another strategy to increase range. For example, acrylamide porous nanoparticles can be loaded with Oregon Grenn ( $\left.p K_{a} 4.1\right)$, fluorescein ( $K_{\mathrm{a}}$ 6.0) and Rhodamine B [63].

Zhou et al [64] have used a different approach by producing a set of on/off fluorescent nanoparticles each with a different emission wavelength and sharp $(<0.25 \mathrm{pH}$ units) $\mathrm{pH}$ transition point, however implementation of this scheme is more complex since a number of different nanoparticles need to be synthesized.

Two-photon fluorescence, which offers the advantage of reduced optical scattering and reduced cellular auto-fluorescence, has also been used in combination with 
nanoparticle-based transduction of $\mathrm{pH}$. Silver nanoparticles coated with 8hydroxypyrene-1,3,6-trisulfonic acid (HPTS) were encapsulated with polyacrylamide and endocytosed by MDA-MB-435 cancer cells [65]. They were then excited at two wavelengths $(900 \mathrm{~nm}$ and $740 \mathrm{~nm})$ and the $\mathrm{pH}$ value was calculated from the ratio of the two fluorescence intensities. A precision of $\sim 0.2 \mathrm{pH}$ units was reported.

Another approach to avoid auto-fluorescence involves the use of fluorescence lifetime imaging microscopy (FLIM) where the image channels represent fluorescence lifetimes rather than the wavelengths and intensities. Cellular autofluorescence has a shorter lifetime (2 to 3 ns) than QDs (five to hundreds of ns) so with time-resolved spectroscopy the signals are easily distinguished. This was exploited by Orte et al [66] who used CdSe/ZnS nanoparticles capped with mercaptoproprionic acid (which have a pH-dependent fluorescence lifetime) to map changes in $\mathrm{pH}$ of endocytic vesicles of cells.

SERS has also been used to map the $\mathrm{pH}$ on a subcellular level. 4-Mercaptobenzoic acid (pMBA) was attached to gold nano-aggregates. The NIH/3T3 cells took up the nanoparticles by endocytosis and the cellular $\mathrm{pH}$ was monitored over 4.5 hours. The $\mathrm{pH}$ was determined to be in the range 6.2 to 6.9 when the nanoparticles were in early stage endosomes, but the range increased to 5 to 6.9 after 4.5 hours due to a variety of endosomes of different ages being present [67]. Large $(0.5-1 \mu \mathrm{m})$ "nanopeapods" of silica nanotubes containing chains of pMBA-capped Au NPs have also been used as SERS intracellular pH transducers [68].

Although endosomal $\mathrm{pH}$ has attracted substantial interest, it is of course also possible to use nanoparticles to probe the $\mathrm{pH}$ in other parts of a cell. For example, Vemula et al encapsulated pHrodo $^{\mathrm{TM}}$ dye with self-assembled hydrogel nanofibers 
[69] and used these to determine the $\mathrm{pH}$ in the cytoplasm, phagosomes and nucleus. C-dots should penetrate even more readily than nanofibers as their very small size facilitates their diffusion into all regions of the cell (see Box 3). Shi et al [70] used dual-labeled (FITC/RITC) C-dots excited at $488 \mathrm{~nm}$ to map the $\mathrm{pH}$ in both cytoplasmic and nuclear regions of HeLa cells treated with oxidizing reagents. The measurable $\mathrm{pH}$ range was 6.0 to 8.0 with an uncertainty of $\sim 0.2 \mathrm{pH}$ units. Very small (2 - $7 \mathrm{~nm}$ ) SiC nanoparticles have been used to map the cytosolic $\mathrm{pH}$ in HeLa cells [71].

\section{Concluding remarks and future perspectives}

Intracellular sensing using nanoparticle-based transduction is a rapidly developing field. Significant progress has been made with mapping the concentration of reactive oxygen species and key biomolecules such as $\mathrm{NADH}$. Temperature and $\mathrm{pH}$ can also be mapped. However, the field is still relatively young and other possibilities have yet to be explored in any detail. Intracellular viscosity, for example, can be deduced from the rotation of a non-spherical magnetic particle within the intercellular environment [72]. Could the force required to penetrate an internal membrane be determined using a magnetic particle? What other possibilities are there? There is also the important question of whether the measurement perturbs the very system that is being studied. The effect of intracellular nanoparticles on cellular processes is an active but as yet still unsettled field. Many nanoparticles exert quite marked effects, often being cytotoxic or damaging in some way. Others, such as GNPs $>5 \mathrm{~nm}$ in size seem to be practically inert. Nanodiamonds also seem to be biocompatible. Effects external to the nanoparticle itself need to be considered. The addition of $\mathrm{Cu}^{2+}$ ions in 
the NADH assay using Au@Cu nanoparticles, for example, might be expected to exert some influence on cellular processes. Despite these difficulties, the scientific insights to be gained from improved methods of quantitatively transducting aspects of the intracellular environment are potentially immense. Nanoparticle-based techniques are already a useful member of the tool-kit that is available, and many exciting new developments in this field may be anticipated.

\section{Acknowledgement}

The authors thank colleagues A. McDonagh, S. Ting and C. Cortie (University of Wollongong) for useful suggestions.

\section{References}

1. Murray, T.V.A., et al. (2014) Reactive oxygen at the heart of metabolism. Trends Cardiovascular Med. 24, 113-120

2. Uusitalo, L.M., and Hempel, N. (2012) Recent advances in intracellular and in vivo ROS sensing: focus on nanoparticle and nanotube applications. Int. J. Mol. Sci. 13, 10660-10679

3. Ayer, A., et al. (2014) Cellular redox homeostasis, reactive oxygen species and replicative ageing in Saccharomyces cerevisiae. FEMS Yeast Research 14, 60-72

4. Pissuwan, D., et al. (2014) Distribution of label free cationic polymer-coated gold nanorods in live macrophage cells reveals formation of groups of intracellular SERS signals of probe nanoparticles. RSC Adv. 4, 5536-5541

5. Gratton, S.E.A., et al. (2008) The effect of particle design on cellular internalization pathways. Proc. Nat. Acad. Sci. 105, 11613-11618

6. Shiao-Wen Tsai, et al. (2013) Internalized gold nanoparticles do not affect the osteogenesis and apoptosis of MG63 osteoblast-like cells: A quantitative, in vitro study. PLoS One 8, e76545

7. Pissuwan, D., et al. (2013) Effect of surface-modified gold nanorods on the inflammatory cytokine response in macrophage cells. Part. Part. Syst. Charact. 30, 427-433

8. Fan, M., and Brolo, A.G. (2009) Silver nanoparticles self assembly as SERS substrates with near single molecule detection limit. Phys. Chem. Chem. Phys. 11, 7381-7389

9. Vitol, E.A., et al. (2012) Nanoprobes for intracellular and single cell surface-enhanced Raman spectroscopy (SERS). J. Raman Spectrosc. 43, 817-827

10. Yuan, H., et al. (2013) Plasmonic nanoprobes for intracellular sensing and imaging. Anal. Bioanal. Chem. 405, 6165-6180 
11. Tsuji, T., et al. (2013) Cationic fluorescent polymeric thermometers with the ability to enter yeast and mammalian cells for practical intracellular temperature measurements. Anal. Chem. 85, 9815-9823

12. Wang, C., et al. (2011) Determining intracellular temperature at single-cell level by a novel thermocouple method. Cell Res. 21, 1517-1519

13. Zhao, X., et al. (2004) A rapid bioassay for single bacterial cell quantitation using bioconjugated nanoparticles. P. Natl. Acad. Sci. 101, 15027-15032

14. Inomata, N., et al. (2012) Pico calorimeter for detection of heat produced in an individual brown fat cell. Appl. Phys. Lett. 100, 154104

15. Gao, L., et al. (2013) Single-cell photoacoustic thermometry. J. Biomed. Opt. 18, 026003

16. Donner, J.S., et al. (2012) Mapping intracellular temperature using green fluorescent protein. Nano Lett. 12, 2107-2111

17. McCabe, K.M., et al. (2011) Lacl(Ts)-regulated expression as an in situ intracellular biomolecular thermometer. Appl. Environ. Microbiol. 77, 2863-2868

18. Yang, J.-M., et al. (2011) Quantum dot nano thermometers reveal heterogeneous local thermogenesis in living cells. ACS Nano 5, 5067-5071

19. Oyama, K., et al. (2012) Walking nanothermometers: spatiotemporal temperature measurement of transported acidic organelles in single living cells. Lab Chip 12, 1591-1593

20. Kucsko, G., et al. (2013) Nanometre-scale thermometry in a living cell. Nature 500, 54-59

21. Albers, A.E., et al. (2012) Dual-emitting quantum dot/quantum rod-based nanothermometers with enhanced response and sensitivity in live cells. J. Am Chem. Soc. 134, 9565-9568

22. Okabe, K., et al. (2012) Intracellular temperature mapping with a fluorescent polymeric thermometer and fluorescence lifetime imaging microscopy. Nat. Commun. 3, 705

23. Nielsen, L.J., et al. (2010) Aptamers Embedded in Polyacrylamide Nanoparticles: A Tool for in Vivo Metabolite Sensing. Acs Nano 4, 4361-4370

24. Zhao, L., et al. (2014) Yolk-Shell Upconversion Nanocomposites for LRET Sensing of Cysteine/Homocysteine. ACS Applied Materials \& Interfaces 6, 11190-11197

25. Ma, W., et al. (2013) Ubiquinone-quantum dot bioconjugates for in vitro and intracellular complex I sensing. Scientific Reports 3, 8

26. Wang, X., et al. (2013) Imaging ROS signaling in cells and animals. J. Mol. Med. 91, 917-927

27. Woolley, J.F., et al. (2013) Recent advances in reactive oxygen species measurement in biological systems. Trends Biochem. Sci. 38, 556-565

28. Hoebe, R.A., et al. (2007) Controlled light-exposure microscopy reduces photobleaching and phototoxicity in fluorescence live-cell imaging. Nat. Biotech. 25, 249-253

29. Hammond, V.J., et al. (2007) An optical sensor for reactive oxygen species: encapsulation of functionalised silica nanoparticles into silicate nanoprobes to reduce fluorophore leaching. Analyst $133,71-75$

30. Kim, H., et al. (2014) ROS-responsive activatable photosensitizing agent for imaging and photodynamic therapy of activated macrophages. Theranostics 4, 1-11

31. Girish, K.S., and Kemparaju, K. (2007) The magic glue hyaluronan and its eraser hyaluronidase: A biological overview. Life Sci. 80, 1921-1943

32. Šoltés, L., et al. (2006) Degradative action of reactive oxygen species on hyaluronan. Biomacromolecules 7, 659-668

33. Lee, H., et al. (2009) Fluorescent gold nanoprobe sensitive to intracellular reactive oxygen species. Adv. Func. Mater. 19, 1884-1890

34. Shiang, Y.-C., et al. (2009) Gold nanodot-based luminescent sensor for the detection of hydrogen peroxide and glucose. Chem. Comm., 3437-3439

35. Auchinvole, C.A.R., et al. (2011) Monitoring intracellular redox potential changes using SERS nanosensors. ACS Nano 6, 888-896 
36. Dmitriev, R.I., and Papkovsky, D.B. (2012) Optical probes and techniques for $\mathrm{O}_{2}$ measurement in live cells and tissue. Cell. Mol. Life Sci. 69, 2025-2039

37. Lee, Y.-E.K., et al. (2009) Nanoparticle PEBBLE sensors in live cells and in vivo. Annu. Rev. Anal. Chem. 2, 57-76

38. Liu, H., et al. (2013) Development of polymeric nanoprobes with improved lifetime dynamic range and stability for intracellular oxygen sensing. Small 9, 2639-2648

39. Kondrashina, A.V., et al. (2012) A phosphorescent nanoparticle-based probe for sensing and imaging of (intra)cellular oxygen in multiple detection modalities. Adv. Func. Mater. 22, 4931-4939

40. Heikal, A.A. (2010) Intracellular coenzymes as natural biomarkers for metabolic activities and mitochondrial anomalies. Biomarkers in Medicine 4, 241-263

41. Zhao, Y., et al. (2011) Genetically encoded fluorescent sensors for intracellular NADH detection. Cell Metab. 14, 555-566

42. Zhang, L., et al. (2011) Single gold nanoparticles as real-time optical probes for the detection of NADH-dependent intracellular metabolic enzymatic pathways. Angew. Chem. Int. Ed. 50, 67896792

43. Freeman, R., et al. (2009) Biosensing and probing of intracellular metabolic pathways by $\mathrm{NADH}$-sensitive quantum dots. Angew. Chem. Int. Ed. Engl. 48, 309-313

44. Meng, Q.T., et al. (2011) Dye-functional mesoporous silica material for fluorimetric detection of $\mathrm{Cr}(\mathrm{III})$ in aqueous solution and biological imaging in living systems. Talanta 86, 408-414

45. Wu, W., et al. (2012) A multifunctional nanosensor based on silica nanoparticles and biological applications in living cells. Chem. Comm. 48, 11017-11019

46. Liu, H., et al. (2013) $\mathrm{Al}^{3+}$-induced far-red fluorescence enhancement of conjugated polymer nanoparticles and its application in live cell imaging. Nanoscale 5, 9340-9347

47. Wu, P.W., et al. (2013) A DNAzyme-Gold Nanoparticle Probe for Uranyl Ion in Living Cells. Journal of the American Chemical Society 135, 5254-5257

48. He, C., et al. (2010) Ratiometric and reusable fluorescent nanoparticles for $\mathrm{Zn}^{2+}$ and $\mathrm{H}_{2} \mathrm{PO}_{4}$ detection in aqueous solution and living cells. J. Mater. Chem. 20, 10755-10764

49. Durgadas, C.V., et al. (2011) Fluorescent gold clusters as nanosensors for copper ions in live cells. Analyst 136, 933-940

50. Lee, H.Y., et al. (2010) BODIPY-functionalized gold nanoparticles as a selective fluorochromogenic chemosensor for imaging $\mathrm{Cu}^{2+}$ in living cells. Analyst 135, 2022-2027

51. Seo, S., et al. (2010) Fluorescein-functionalized silica nanoparticles as a selective fluorogenic chemosensor for $\mathrm{Cu}^{2+}$ in living cells. Eur. J. Inorg. Chem., 843-847

52. Liu, X., et al. (2014) Carbon dots based dual-emission silica nanoparticles as a ratiometric nanosensor for $\mathrm{Cu}^{2+}$. Anal. Chem. 86, 2289-2296

53. Zong, C.H., et al. (2011) Dual-emission fluorescent silica nanoparticle-based probe for ultrasensitive detection of $\mathrm{Cu}^{2+}$. Anal. Chem. 83, 3126-3132

54. Zhu, A.W., et al. (2012) Carbon-dot-based dual-emission nanohybrid produces a ratiometric fluorescent sensor for in vivo imaging of cellular copper ions. Angew. Chem. Int. Ed. 51, 7185-7189

55. Si, D., et al. (2012) Nanoparticle PEBBLE sensors for quantitative nanomolar imaging of intracellular free calcium ions. Anal. Chem. 84, 978-986

56. Ray, A., et al. (2011) Two-photon nano-PEBBLE sensors: subcellular pH measurements. Analyst 136, 3616-3622

57. Wencel, D., et al. (2014) Optical chemical pH sensors. Anal. Chem. 86, 15-29

58. Madsen, J., et al. (2013) Nile Blue-based nanosized pH sensors for simultaneous far-red and near-infrared live bioimaging. J. Am Chem. Soc. 135, 14863-14870

59. Kay, E.R., et al. (2013) Conformational control of energy transfer: a mechanism for biocompatible nanocrystal-based sensors. Angew. Chem. Int. Ed. 52, 1165-1169

60. Chen, Y.P., et al. (2012) Surface charge effect in intracellular localization of mesoporous silica nanoparticles as probed by fluorescent ratiometric $\mathrm{pH}$ imaging. RSC Adv. 2, 968-973 
61. Tsou, C.J., et al. (2013) A broad range fluorescent pH sensor based on hollow mesoporous silica nanoparticles, utilising the surface curvature effect. J.Mater. Chem. B 1, 5557-5563

62. Ho, L.C., et al. (2013) Sensitive pH probes of retro-self-quenching fluorescent nanoparticles. J. Mater. Chem. B 1, 2425-2432

63. Benjaminsen, R.V., et al. (2011) Evaluating nanoparticle sensor design for intracellular pH measurements. ACS Nano 5, 5864-5873

64. Zhou, K.J., et al. (2012) Multicolored pH-tunable and activatable fluorescence nanoplatform responsive to physiologic pH stimuli. J. Am Chem. Soc. 134, 7803-7811

65. Ray, A., et al. (2012) Two-photon fluorescence imaging super-enhanced by multishell nanophotonic particles, with application to subcellular pH. Small 8, 2213-2221

66. Orte, A., et al. (2013) Fluorescence lifetime imaging microscopy for the detection of intracellular $\mathrm{pH}$ with quantum dot nanosensors. ACS Nano 7, 6387-6395

67. Kneipp, J., et al. (2010) Following the dynamics of pH in endosomes of live cells with SERS nanosensors. J. Phys. Chem. C 114, 7421-7426

68. Cong, V.T., et al. (2014) Gold Nanoparticle Silica Nanopeapods. Journal of the American Chemical Society 136, 3833-3841

69. Vemula, P.K., et al. (2014) Self-assembled hydrogel fibers for sensing the multi-compartment intracellular milieu. Sci. Rep. 4, 9

70. Shi, W., et al. (2012) A tunable ratiometric pH sensor based on carbon nanodots for the quantitative measurement of the intracellular pH of whole cells. Angew. Chem. Int. Ed. 51, 64326435

71. Guo, J.H., et al. (2013) In situ probing of intracellular pH by fluorescence from inorganic nanoparticles. Biomaterials 34, 9183-9189

72. McNaughton, B.H., et al. (2007) Physiochemical microparticle sensors based on nonlinear magnetic oscillations. Sens. Actuators B 121, 330-340 


\section{Glossary}

Biosensor: is a device for detection and quantification of small biologicallyrelevant molecules. It interacts with the biochemical and this stimulus is transduced to, for example, an optical signal.

Endocytosis: is a process by which a cell absorbs large molecules or nanoparticles by engulfing them

Endosomes: are intracellular vesicles into which the foreign matter absorbed by endocytosis are initially placed

Hyaluronic acid (HA): is a carbohydrate polymer that is common in tissues and the extracellular matrix

Nicotinamide adenine dinucleotide (NADH): is a metabolic coenzyme that plays an important role in cell activity. Forms a redox couple with its oxidized form $N A D^{+}$.

Reactive oxygen species (ROS): are reactive molecules or free radicals such as $\mathrm{H}_{2} \mathrm{O}_{2}, \mathrm{O}_{2}{ }^{-}$or $\cdot \mathrm{OH}$. These play an important role in living cells. They are generated naturally by cellular metabolic processes and have a potent effect on signaling, DNA, proteins, lipids, redox homeostasis, metabolism and aging [1-3]

Transduction: is the process of converting a signal from one form (e.g. chemical, magnetic, temperature) to another (e.g. electrical, optical). 


\section{Box 1. Quantum dots, gold nanoparticles, and fluorophores}

A quantum dot is an inorganic semiconductor nanoparticle, typically less than $10 \mathrm{~nm}$ in size. Examples include CdSe@ZnS (a CdSe core surrounded by a ZnS shell). These are used as fluorescent probes and are characterised by narrow emission lines, the wavelength of which can be controlled by the nanoparticle size. They are brighter than organic dyes and do not photobleach, however they do have disadvantages such as potential cytotoxicity and fluorescence blinking. Carbon dots (C-dots) are recently developed platform showing great potential for biosensing applications because of their biocompatibility and versatile surface chemistry.

Gold nanoparticles (GNPs) of between 5 to $50 \mathrm{~nm}$ in diameter undergo a strong electromagnetic resonance with light of wavelengths of the order of 520 to 550 $\mathrm{nm}$. This resonance causes the electric field in the vicinity of the particle to be greatly enhanced. If a fluorophore (QD or dye molecule) is located within 20 or 30 $\mathrm{nm}$ of the GNP then its light emission will be attenuated. Essentially, the energy of the excitation within the fluorophore is transferred into the GNP by a dipole-todipole interaction. The situation is illustrated schematically below, in which an $\mathrm{SiO}_{2}$ spacer has been used to provide a reproducible separation between a GNP and a dye molecule. (Reprinted with permission from Reineck et al, ACS Nano, vol.7, 2013, pp.6636-6648. Copyright (2013) American Chemical Society.)

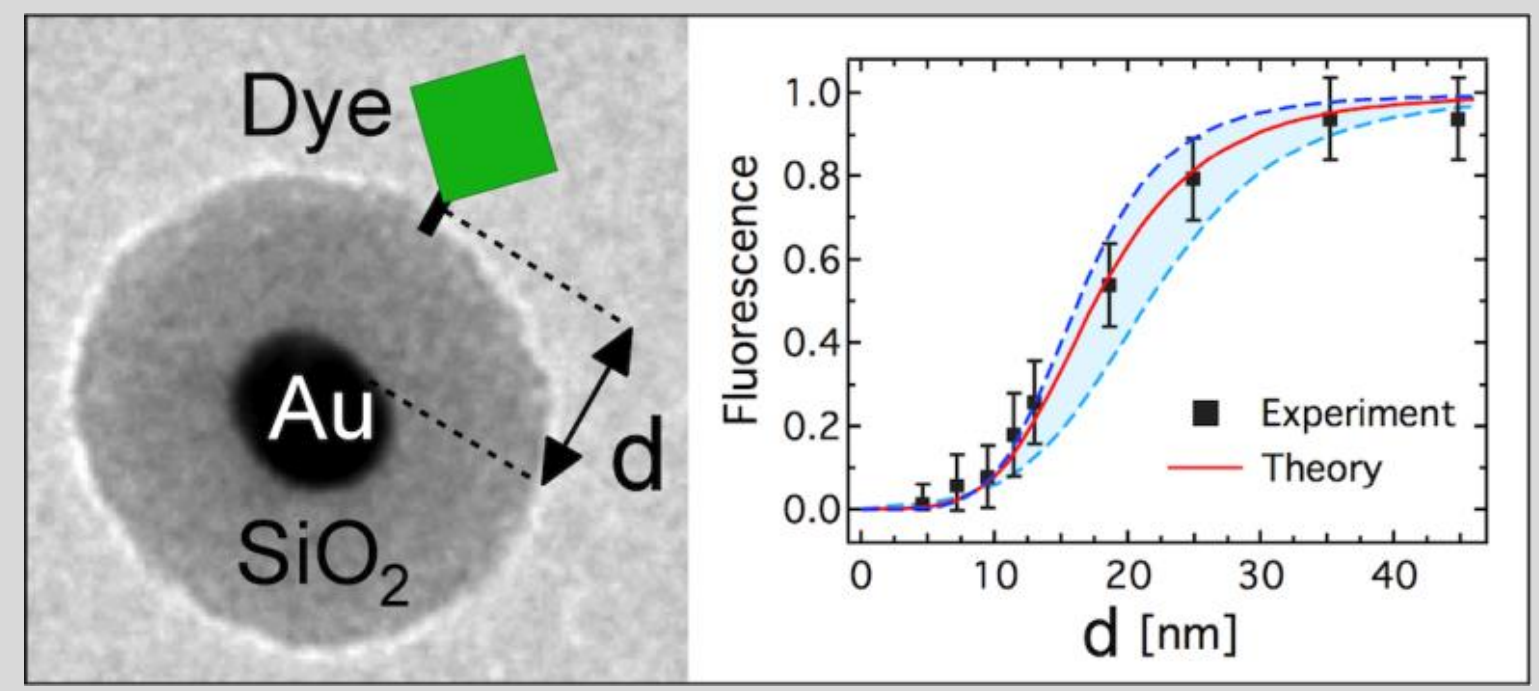




\section{Box 2. Imaging Methodologies}

FRET (Förster resonance energy transfer) - This effect transduces the distance $r$ between donor and acceptor fluorophores since the efficiency depends on $r^{6}$. It is effective when the analyte causes a displacement between donor and acceptor moieties or cleaves one moiety altogether. When FRET takes place an excited fluorescent donor relaxes to its ground state transferring energy to an acceptor via dipole-dipole coupling. An increase in transfer efficiency results in quenching of donor fluorescence and increase in acceptor fluorescence if the acceptor is fluorescent.

Ratiometric Method - This method is based on the ratio of two independent fluorescence intensities. The analyte interacts with one fluorophore to change its fluorescence yield while a second fluorophore is insensitive to the presence of the analyte. This technique has the advantage of automatically taking into account variations in excitation intensity, local nanoparticle concentration etc.

SERS (surface enhanced Raman scattering) - Raman spectroscopy yields the vibrational energies of molecules which can be used to identify the molecule or its conformation. In the SERS effect the Raman signal of a molecule in the immediate proximity of a metal nanoparticle is enhanced by a factor $10^{4}-10^{12}$ and background fluorescence may also be quenched.Gold or silver substrates are typically used. In the case of a biosensor, the molecule which undergoes enhanced scattering may be the analyte itself, or more commonly, a reporter molecule whose vibrational levels change when it interacts with the analyte. 


\section{Box 3. Outstanding questions}

Nanoparticles most commonly enter the cell through the endocytic pathway and remain localised in endosomes. What are the best ways to deliver nanoparticles to other parts of the cell, such as the cytosol or nucleus, for extended quantitative analysis and imaging?

Mitochondria are of particular interest as they produce most of the cell's ROS and heat, consume most of its oxygen and have a pH which varies widely across their cross-sections. What options are there for targeting the nanoparticles for specific organelles like the mitochondria, which are relatively resistant to penetration by any kind of probe?

Some nanoparticles have cytotoxic effects, but even seemingly inert nanoparticles can modify cellular behaviour. To what extent do various nanoparticle transducers perturb the processes within the target region of the cell in any significant manner and how can this be mitigated? 


\section{Figure Captions}
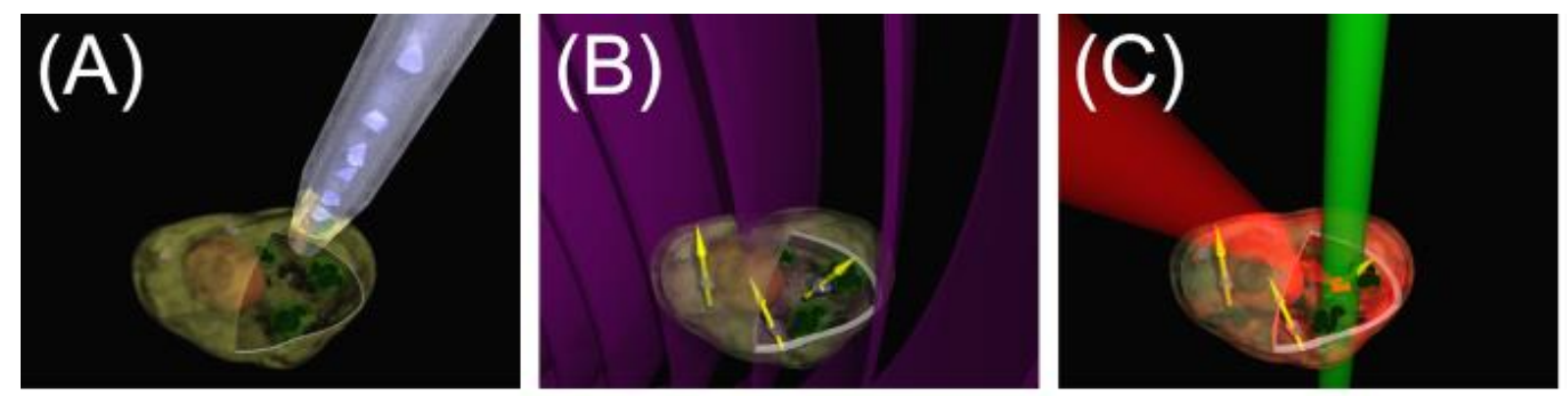

Figure 1. Use of nanodiamonds as ultrasensitive temperature sensors in live cells. (A) Principles of the process: (i) and (ii) cell is injected with several nano-diamonds, (iii) electronic spin (yellow arrows) of defect sites in nano-diamonds excited using microwave radiation, (iv) temperature-dependent spin state read-out by analyzing fluorescent emission (red) of selected nano-diamond using probe laser (green). (B) Temperature indicated as fluorescence counts per second (c.p.s). The cell has been heated using the plasmon resonance of a gold nanoparticle located at X. (C) Calibration of thermometer. (Panels B and C reprinted by permission from Macmillan Publishers Ltd: G. Kucsko, P. C. Maurer, N. Y. Yao, M. Kubo, H. J. Noh, P. K. Lo, H. Park \& M. D. Lukin, Nanometre-scale thermometry in a living cell. Nature, vol.500, 2013, pp.54-59, copyright (2013).) 


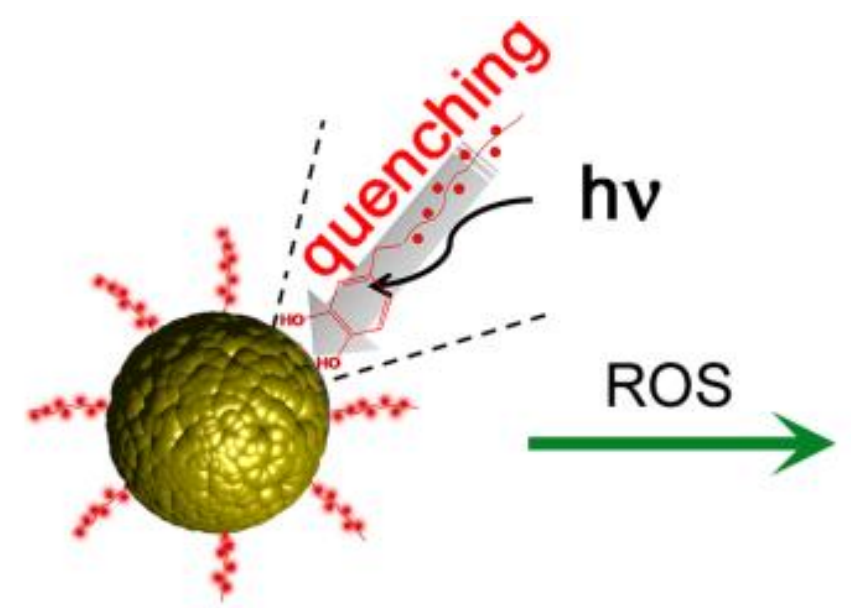

Fluorescence off

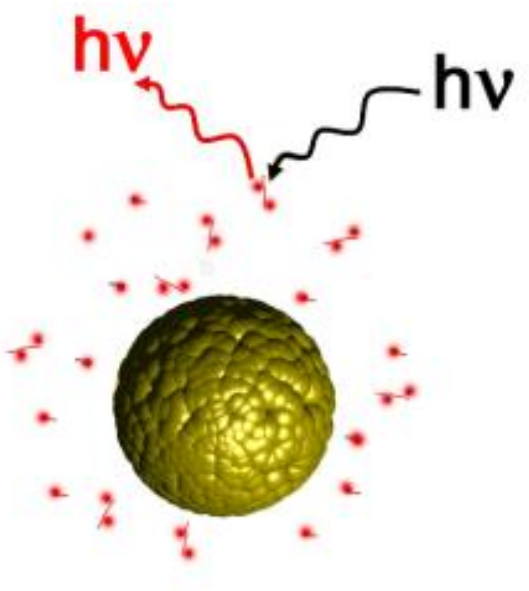

Fluorescence on

Figure 2. (A) Schematic illustration of GNP-probe immobilized with fluorescein-HA conjugates for intracellular ROS monitoring, (i) when dye is attached to GNP its fluorescence is quenched, (ii) presence of ROS cleaves dye from GNP enabling fluoresence. (B) Monitoring of intracellular ROS generation for $2 \mathrm{~h}$ in macrophages stimulated with LPS and then treated with functionalized gold nanoparticles. The production of ROS was indicated by green fluorescence. The cell nuclei are stained blue in these images. Redrawn with permission from Lee et al. [33]. 
(A)

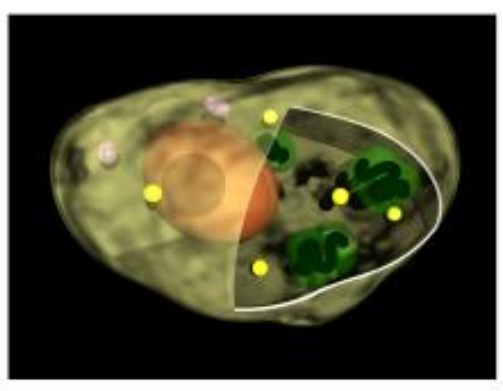

(B)

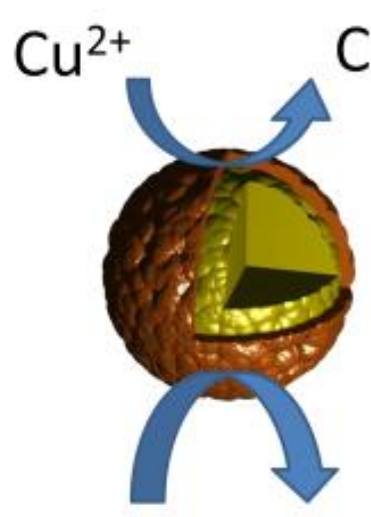

$\mathrm{NADH} \mathrm{NAD}^{+}$

$\mathrm{Cu}^{0}$
(C)

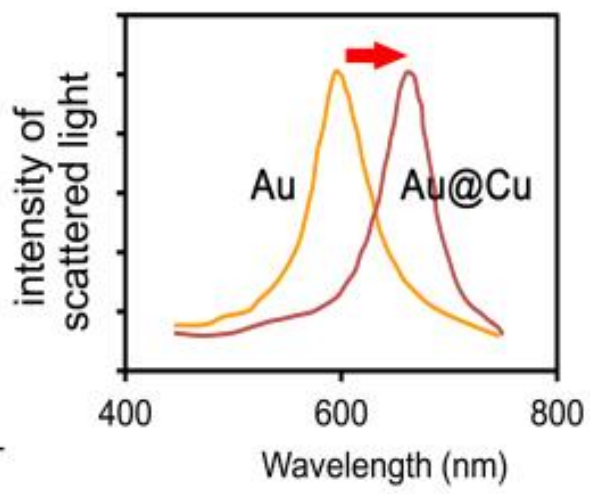

Figure 3. Reduction of $\mathrm{a} \mathrm{Cu}^{0}$ shell onto a Au nanoparticle core is a quantitative measure of NADH. (A) Principle of process: (i) gold nanoparticles are introduced into cell, (ii) NADH in cell will reduce $\mathrm{Cu}^{2+}$ to $\mathrm{Cu}$ to form a shell on the $\mathrm{Au}$, (iii) formation of the $\mathrm{Cu}$ shell causes a marked red-shift on the scattering spectrum. (B) (iv) HeLa cells incubated with gold nanoparticles and $\mathrm{CuCl}_{2}(v)$ Au@Cu nanoparticles scatter light in the dark-field optical image of the previous panel, ( $v i)$ spectra are collected of individual particles (circled) and their redshift determined. Panel (B) reproduced with permission from Zhang et al. [42] (with some lines removed for clarity). 